\title{
Eikonal particle scattering and dilaton gravity
}

\author{
Saurya Das and Parthasarathi Majumdar* \\ The Institute of Mathematical Sciences, CIT Campus, Madras - 600 113, India
}

(Received 31 July 1996)

\begin{abstract}
Approximating light charged pointlike particles in terms of (nonextremal) dilatonic black holes is shown to lead to certain pathologies in Planckian scattering in the eikonal approximation, which are traced to the presence of a (naked) curvature singularity in the metric of these black holes. The existence of such pathologies is confirmed by analyzing the problem in an "external metric" formulation where an ultrarelativistic point particle scatters off a dilatonic black hole geometry at large impact parameters. The maladies disappear almost trivially upon imposing the extremal limit. Attempts to derive an effective three-dimensional "boundary" field theory in the eikonal limit are stymied by four-dimensional (bulk) terms proportional to the light-cone derivatives of the dilaton field, leading to nontrivial mixing of electromagnetic and gravitational effects, in contrast with the case of general relativity. An eikonal scattering amplitude, showing decoupling of these effects, is shown to be derivable by resummation of graviton, dilaton, and photon exchange ladder diagrams in a linearized version of the theory for an asymptotic value of the dilaton field which makes the string coupling constant nonperturbative. [S0556-2821(97)00104-5]
\end{abstract}

PACS number(s): 04.62.+v, 04.50.+h, 11.80.Et, 11.80.Fv

\section{INTRODUCTION}

Nontrivial nonperturbative information regarding gravitational interactions is now well known to be accessible via point particle scattering in four-dimensional Minkowski space at Planckian center-of-mass energies and fixed, low momentum transfers $[1,2]$. The singular kinematics of this (eikonal) approximation lead to a truncated dynamics amenable to exact treatment without further approximations. The easiest way to visualize these collision processes is through the shock wave picture [1,3]: an ultrarelativistic point particle produces a background with the geometry of two Minkowski spacetimes glued together after a shift along the null direction (in Minkowski space) characterizing the motion of the particle [4]. The other null direction can be taken to define the affine parameter for the null geodesic of a test particle encountering this shock wave geometry. The quantum mechanical amplitude of this collision is exactly calculable, so long as $G s \approx 1$, where $G$ is Newton's constant. A field theoretic analysis reproduces identical results for the amplitudes while yielding a reduced three-dimensional field theory which describes the suppression of standard graviton exchanges relative to the instantaneous interaction mediated by the shock wave [5]. Leading order corrections to the eikonal process have also been computed using superstring theory in the Regge-Gribov formalism [2].

The inclusion of and interplay (of gravitation) with electromagnetism, in this kinematical domain, has also been investigated in detail [6-10], incorporating situations where the particles may have both electric and magnetic charge. In so far as general relativity is concerned, some remarkable phenomena occur in the eikonal region: electromagnetic and gravitational interactions seem to operate quite independently of each other, in contrast to more generic kinematical

*Electronic address: saurya,partha@imsc.ernet.in situations [8]. ${ }^{1}$ Also, while gravitational interactions characterized by the dimensionless quantity $G s$ are usually taken to dominate in this region ( $G s \approx 1$ ), compared to electromagnetism, which is controlled by $\alpha \approx 1 / 137$ for small momentum transfers, with magnetic charges present this is no longer the case [7].

The variant of general relativity known as dilaton gravity is an important extension of the standard Einstein theory because it appears in the low energy approximation to superstring theory [11]. The behavior of dilaton gravity in the kinematics of the eikonal approximation is a question of intrinsic interest vis-à-vis the simplifications mentioned above. On somewhat heuristic grounds, it has been shown [8] that the decoupling of gravity and electromagnetism seen earlier may not actually occur for the case of dilaton gravity, owing primarily to the coupling of the dilaton field to the metric (or to the electromagnetic field strength). In this paper we turn to a more comprehensive analysis of dilaton gravity in the eikonal domain, to see if these heuristic results may indeed have a firmer basis. Thus, if the particles in question are approximated in their static limit by charged dilatonic black holes, then is the geometry due to such a particle similar to a gravitational shock wave when the particle moves almost luminally? The issue of the eikonal scattering amplitude in this case is an immediate consequence. The reduction of the full set of degrees of freedom to a truncated set amenable to exact mathematical treatment is another issue of importance that must be addressed.

The paper is organized as follows: in Sec. II we review our earlier work using the boosting techniques of Ref. [4] to examine the interplay of gravity and electromagnetism. We further demonstrate how the problems discerned might dis-

\footnotetext{
${ }^{1}$ This has been further confirmed in an independent analysis using the external metric formulation of the problem, wherein an almost luminal particle scatters off the static metric of a charged (ReissnerNordström) black hole [10].
} 
appear in the extremal limit. In Sec. III, both the nonextremal and the extremal situations are reanalyzed within the external (static dilatonic black hole) metric formalism; in the former case, we show how it is impossible to reduce the equation of motion of an ultrarelativistic particle in this metric to a solvable Schrödinger-like form useful for extracting phase shifts. Once again, the pathology is obviated in the extremal limit wherein solutions identical to those in a Schwarzschild background [10] ensue. In Sec. IV, we turn to a field theoretic analysis following [5], wherein we point out the difficulties of reducing the theory in the relevant kinematical domain to a boundary field theory which "lives" in a threedimensional space composed by the transverse twodimensional plane and the boundary of the null plane. This concomitantly demonstrates the nontrivial mixing of gravitational and electromagnetic interactions in this case. Section $\mathrm{V}$ probes the possibility of a derivation of the quantum eikonal amplitude by resummation of ladder-type exchange graphs in a linearized version of the theory. The linearization is argued to be invalid in the regime of perturbative string coupling. We conclude in Sec. VI with a few remarks on what our results might indicate from a string theoretic standpoint.

\section{DILATON GRAVITY HEURISTICS}

This section is a brief review of our earlier work [8]. We begin by considering the static, spherically symmetric and electrically charged solution of dilaton gravity in the socalled "string metric" [12], which is a solution of the low energy string effective action:

$$
\begin{aligned}
d s^{2}= & \left(1-\frac{\alpha}{M r}\right)^{-1}\left[\left(1-\frac{2 G M}{r}\right) d t^{2}-\left(1-\frac{2 G M}{r}\right)^{-1} d r^{2}\right. \\
& \left.-\left(1-\frac{\alpha}{M r}\right) r^{2} d \Omega^{2}\right] .
\end{aligned}
$$

Here $\alpha \equiv Q^{2} e^{2 \phi_{0}}, Q$ being the electric charge and $\phi_{0}$ the asymptotic value of the dilaton field. We confine ourselves to situations not subject to the extremality condition $Q^{2} e^{2 \phi_{0}}=2 G M^{2}$. It may be noted that this metric differs from the Reissner-Nordström solution of general relativity in that it does not have two horizons, while it has a curvature singularity at $r=\alpha / M$. This difference is due to the presence of the dilaton field. As this metric describes the spacetime around a point particle of mass $M$, to obtain the same when the particle is massless and travels along the null geodesic $x^{-} \equiv t-z=0$, we boost this metric along the positive $z$ axis to a velocity $\beta$ and take the limit $\beta \rightarrow 1$. On parametrizing the mass as $M=p / \gamma$, where $\gamma=\left(1-\beta^{2}\right)^{-1 / 2}$ and $p$ is the energy of the particle, and introducing the other light cone coordinate $x^{+}=t+z$, we get [8]

$$
d s^{2} \rightarrow d \widetilde{x}^{+} d \widetilde{x}^{-}-\left(d \widetilde{x_{\perp}}\right)^{2},
$$

where

$$
\begin{gathered}
d \widetilde{x}^{+}=d x^{+}-\left(\begin{array}{c}
\frac{4 G p}{\left|x^{-}\right|} \\
1-\frac{\alpha}{p\left|x^{-}\right|}
\end{array}\right) d x^{-}, \\
d \widetilde{x}^{-}=d x^{-}\left(\begin{array}{c}
1-\frac{\alpha}{2 p\left|x^{-}\right|} \\
1-\frac{\alpha}{p\left|x^{-}\right|}
\end{array}\right), \\
d \overrightarrow{\widetilde{x}}_{\perp}=d \vec{x}_{\perp} .
\end{gathered}
$$

We observe that in addition to the shift in the $x^{+}$coordinate (as for the Schwarzschild metric), the coordinate $\tilde{x}^{-}$, depends on the charge $\alpha$. This is made explicit by choosing $\alpha$ to be small (achieved either by considering a small charge $Q$ or by taking a large negative value of $\phi_{0}$ ). Then the above equations can be linearized to obtain

$$
\begin{gathered}
d \widetilde{x}^{+}=d x^{+}-\frac{4 G p}{\left|x^{-}\right|}-\frac{4 \alpha}{\left(x^{-}\right)^{2}}+O\left(\alpha^{2} / p\right), \\
d \tilde{x}^{-}=d x^{-}+\frac{\alpha}{2 p\left|x^{-}\right|}+O\left(\alpha^{2} / p^{2}\right) .
\end{gathered}
$$

The $\alpha$ dependent shift in $x^{+}$, being a continuous function of $x^{-}$, can be removed by a diffeomorphism while the shift in $x^{-}$cannot, because of the presence of the discontinuous function $\theta\left(x^{-}\right)$. Interestingly, for the Reissner-Nordström metric, the $Q$-dependent piece can also be removed by a diffeomorphism. Now, for a test particle in the background geometry of this right-moving particle, the coordinate $x^{-}$ serves as its affine parameter, and a discontinuity in the latter signals a serious breakdown of the boosting method. Specifically, the interpretation of the boosted metric as two Minkowski spaces glued together at the null plane $x^{-}=0$ after a shift in the coordinate $x^{+}$(cut and paste prescription) is no longer possible as for the Schwarzschild [4] or the Reissner-Nordström [8] metric. This becomes apparent when one writes the classical geodesic equations for a light test particle in the background of the boosted dilaton metric and tries to solve it perturbatively in a power series in the mass $M$ using singular perturbation theory. The failure of the latter indicates that the the null geodesics are incomplete in this case, and curvature singularity at $r=\alpha / M$ shows up as an extended naked singularity in the boosted limit [8]. Thus the geometry is intractably more complicated, which renders a calculation of the corresponding scattering amplitude impossible.

Having confronted the above mentioned difficulty, let us try to see whether the same can be circumvented for certain special values of the parameters. For example, the extremal limit can be considered for its special role in certain other situations (it has zero entropy and Hawking temperature). For the space-time depicted in the metric (1), the extremal limit corresponds to the merging of the Schwarzschild horizon and the sphere of curvature singularity. The condition among the parameters is therefore $\alpha=2 G M^{2}$, which when translated in the expression for the metric yields 


$$
d s^{2}=-d t^{2}+\frac{d r^{2}}{\left(1-\frac{2 G M}{r}\right)^{2}}+r^{2} d \Omega^{2}
$$

On performing the boosting procedure on this, we get

$$
d s^{2}=d x_{\perp}^{2}-d x^{-}\left[d x^{+}-4 G p \frac{d x^{-}}{\left|x^{-}\right|}\right],
$$

which can be seen to coincide with Eq. (4) for $\alpha=0$. Note that this is the same as a boosted Schwarzschild geometry [4], although the metric (5) cannot be identified with a Schwarzschild space-time. In fact, this metric is singularity free and geodesically complete. Since there is a shift in the light cone coordinate $x^{+}$only, the affine parameter $x^{-}$is continuous, and the "cut-and-paste" prescription is eminently applicable. The corresponding scattering amplitude is the well-known eikonal result [1]

$$
f(s, t)=\frac{1}{t} \frac{\Gamma(1-i G s)}{\Gamma(1+i G s)}\left(\frac{1}{-t}\right)^{-i G s},
$$

where $s$ is the square of the center-of-mass energy. It may be noted that the above amplitude refers to gravitational interactions only. In addition, due to charges on the particles, there can be electromagnetic contributions to the scattering. How they affect the latter has been dealt with at length in [8] and [10]. We will briefly touch upon this issue in Sec IV. We will also come back to the issue of taking the extremal limit in the subsequent sections and try to understand why it leads to a reasonable result.

\section{EXTERNAL METRIC APPROACH}

A better physical insight into why such a breakdown occurs for the generic dilaton gravity metric may emerge upon analyzing the above physical situation by a manifestly covariant approach, in which we solve for the wave equation of a test particle in the fixed background space-time created by the other particle. As emphasized earlier, this space-time can be modeled by the dilaton black hole solution as in Eq. (1). For simplicity, we define the quantities

$$
\Lambda=1-\frac{2 G M}{r}
$$

and

$$
\Delta=1-\frac{\alpha}{M r} .
$$

The Klein-Gordon equation of the (spinless) test particle is given by

$$
D_{\mu} D^{\mu} \phi=0,
$$

where $D_{\mu}$ denotes the relativistically covariant derivative in the metric (1). Assuming a solution for $\phi$ of the form

$$
\phi(\vec{r}, t)=\phi(r) Y_{l m}(\theta, \phi) e^{i E t},
$$

(where $E$ is the energy of the test particle as measured by an asymptotic observer) and with the "string" metric (1) in the background, the radial part of Eq. (8) becomes

$$
r^{2} \Lambda \frac{d^{2} \phi(r)}{d r^{2}}+\frac{d\left(r^{2} \Lambda\right)}{d r} \frac{d \phi(r)}{d r}-\left[\frac{l(l+1)}{\Delta}-\frac{E^{2} r^{2}}{\Lambda}\right] \phi(r)=0 .
$$

For generic values of $\Lambda$, the first derivative term can be ignored and on setting $\Delta=1$ (i.e., no dilatonic and/or electric charge), we recover the radial equation of a neutral particle in a Schwarzschild background [8]:

$$
\frac{d^{2} f}{d r^{2}}-\left[\frac{l(l+1)-3(G s)^{2}}{r^{2}}-\frac{2 G s E}{r}-E^{2}\right] f=0 .
$$

Here, $\phi(r)=f(r) / r$. For large $l$ (the eikonal limit), this equation is just the Schrödinger equation for a charge in a Coulomb potential, once we identify the electromagnetic coupling constant $\alpha$ with $\alpha_{G} \equiv G s$ (with a minus sign) and the momentum $k$ with the energy $E$. The subsequent calculation of the scattering phase shifts is exact. The expression for the phase shift is $[14,10]$

$$
\delta_{l}=\arg \Gamma(l+1-i G s) .
$$

The scattering amplitude obtained from this phase shift agrees with Eq. (7). However, we are interested to know whether for generic values of $\Delta$, the above equation reduces to a Schrödinger-like equation, amenable to scattering solutions. In the latter case, $\Delta$ vanishes and the centrifugal term becomes singular at a radius $r=\alpha / M$. In the limit that $M$ is small, this corresponds to very large radial distances. Thus the curvature singularity appears in the vicinity of the test particle trajectory (with fixed large impact parameter $b$ ) and the tacit assumption that the test particle trajectory is in a region of small curvature, fails. This warrants a careful analysis of the radial equation in this region. The coefficient of $\phi(r)$ in Eq. (10) is

$$
p^{(2)} \equiv \frac{E^{2} r^{2}}{\Lambda}-\frac{l(l+1)}{\Delta} .
$$

In the domain of interest $0<r<\infty, p^{(2)}$ fails to be continuous at $r=\alpha / M$. This is because

$$
\begin{aligned}
& \lim _{r \rightarrow(\alpha / M)^{-}} p^{(2)} \rightarrow+\infty, \\
& \lim _{r \rightarrow(\alpha / M)^{+}} p^{(2)} \rightarrow-\infty,
\end{aligned}
$$

and $\left.p^{(2)}\right|_{r=\alpha / M}$ is not defined. An elementary theorem in the theory of ordinary differential equations states that, under these circumstances, a unique solution of Eq. (10) does not exist [13]. Similar conclusions follow by considering the radial equation in the "Einstein" metric, which is related to the string metric by a Weyl transformation of the form $g_{\mu \nu}^{\text {Einstein }}=e^{2 \phi} g_{\mu \nu}^{\text {string }}$. This can be seen by writing the radial equation in this case, which is 


$$
\begin{aligned}
& r^{2} \Lambda \frac{d^{2} \phi(r)}{d r^{2}}+\left[\frac{d\left(r^{2} \Lambda\right)}{d r}+\frac{r^{2} \Lambda}{\Delta} \frac{d \Delta}{d r}\right] \frac{d \phi(r)}{d r} \\
& -\left[\frac{l(l+1)}{\Delta}-\frac{E^{2} r^{2}}{\Lambda}\right] \phi(r)=0 .
\end{aligned}
$$

Here, in addition to $p^{(2)}$, the coefficient $p^{(1)}$ of the first derivative term also becomes discontinuous at $r=\alpha / M$ due to the presence of the additional $\Delta$-dependent piece. So, we can no longer ignore the first derivative term. In any case, a unique solution still does not exist.

Thus we see that, for vanishing particle masses, it is impossible to extract a Schrödinger-like differential equation for the dilaton gravity metric from which we can compute a unique scattering solution and the corresponding phase shift. Basically, the reason is that the factor in the metric incorporating dilaton effects, namely $(1-\alpha / M r)$, blows up as $M \rightarrow 0$, thus rendering the equation analytically intractable. As the particle masses decrease, the location of the curvature singularity of the black hole recedes farther away from the origin $r=0$ without limit. Any particle in the field of this black hole, however large its impact parameter, is trapped within this naked singularity. This is reflected in the nonexistence of well-defined quantum scattering solutions. The gulf of difference between the earlier analyses involving the Schwarzschild and Reissner-Nordström metrics [10] and the present case need hardly be over emphasized. The problem is obviously absent for macroscopic stellar objects with large masses, for which the naked singularity is well hidden behind the event horizon. One can then expand the coefficients of the radial equation involving $\Delta$ in powers of the small parameter $\alpha / M r$ and obtain a perturbative solution. This would yield finite $\alpha$-dependent corrections to the scattering amplitude (7) which, however, detracts from our aim of studying point particle scattering.

Instead, it makes more sense to investigate the extremal limit which was seen to cure the malady in the previous section. Substituting $\Lambda=\Delta$, for the extremal limit in Eq. (10), we get

$\frac{d^{2} \phi(r)}{d r^{2}}+\frac{1}{r^{2} \Lambda} \frac{d\left(r^{2} \Lambda\right)}{d r} \frac{d \phi(r)}{d r}-\frac{1}{\Lambda^{2}}\left[\frac{l(l+1)}{r^{2}}-E^{2}\right] \phi(r)=0$.

Expanding $\Lambda$ in powers of $G M / r$ and retaining terms to the appropriate order, this reduces to the Schwarzschild radial equation (11), and the scattering amplitude is once again Eq. (7). Identical conclusions follow when one uses the Einstein metric instead of the string metric.

\section{SCALING AND BOUNDARY FIELD THEORY}

So far, we have explicitly used the solutions of the dilaton gravity action to model the point particles. In the second section, the boosted particle was regarded as the source in the background of which the slow particle scattered, while the latter served as the source of a static spherically symmetric geometry in Sec. III. In either case, the model failed except in the extremal limit. Now we approach the eikonal limit in a "solution-independent way." In other words, by imposing certain kinematical restrictions, we suitably trun- cate the action of the theory such that it automatically incorporates the eikonal kinematics. An important observation ensues to the effect that all local degrees of freedom decouple from the theory, leaving behind a residual boundary valued action. This has been demonstrated in the case of general relativity and electrodynamics separately in [5] and [6], respectively. Our task would consist of two parts. First, to show that in the Einstein-Maxwell framework, the decoupling of the interactions takes place at the level of the action, as claimed in [8] on the basis of a heuristic analysis. Second, to investigate to what extent similar arguments would hold for the case of the dilaton gravity action. The advantage of this method is that one does not have to resort to explicit classical solutions at all.

We begin with the Einstein action

$$
S_{E}=-\frac{1}{G} \int d^{4} x \sqrt{-g} R \text {. }
$$

On choosing a gauge for the metric tensor such that its longitudinal $(+,-)$ modes are manifestly decoupled from the transverse modes $(i, j)$, and retaining only those configurations which are consistent with the high momenta in the longitudinal direction and low momenta in the transverse direction, the Einstein action reduces to an action on the boundary $\partial M$ of the two-dimensional Minkowski subspace in the form [5]

$$
S_{E} \rightarrow S_{E[\partial M]}=\frac{1}{G} \int \sqrt{g}\left(\sqrt{h} R_{h}+\frac{1}{4} \sqrt{h} h^{i j} \partial_{i} g_{\alpha \beta} \partial_{j} g_{\gamma \delta} \epsilon^{\alpha \gamma} \epsilon^{\beta \delta}\right) .
$$

Here, all quantities pertaining to $g$ (with Greek indices) and $h$ (Latin indices) are related to the longitudinal and transverse subspaces, respectively. The metric components satisfy the constraints

$$
\begin{gathered}
h_{i j}=h_{i j}(x, y), \\
g_{\alpha \beta}=\eta_{a b} \partial_{\alpha} X^{a} \partial_{\beta} X^{b},
\end{gathered}
$$

whereby $h_{i j}$ is no longer a propagating degree of freedom, and $g_{\alpha \beta}$ is conformally flat up to diffeomorphisms of the longitudinal subspace. Thus, only the boundary values of the diffeomorphism parameter $X^{a}$ remain as the surviving dynamical degrees of freedom in the eikonal limit.

The corresponding electromagnetic action in flat space, namely,

$$
S_{\mathrm{EM}}=-\frac{1}{4} \int d^{4} x F_{\mu \nu} F^{\mu \nu}
$$

truncates (in the Lorentz gauge) to [6]

$$
\begin{aligned}
S_{\mathrm{EM}} \rightarrow S_{\mathrm{EM}[\partial M]}= & \oint d \tau \int d^{2} r_{\perp}\left(\frac{1}{2} \Omega^{-} \nabla^{2} \partial_{\tau} \Omega^{+}\right. \\
& \left.-\frac{1}{2} \Omega^{+} \nabla^{2} \partial_{\tau} \Omega^{-}\right),
\end{aligned}
$$

with the constraints for the fields: 
$F_{ \pm}=0 ; \quad A_{ \pm}=\partial_{ \pm} \Omega ; \quad \Omega(x)=\Omega^{+}\left(x^{+}, \vec{r}_{\perp}\right)+\Omega^{-}\left(x^{-}, \vec{r}_{\perp}\right)$.

$A_{i}$ is a classical background and can be taken to be zero without loss of generality. For both the gravity and electromagnetic actions, it can be shown that the addition of the terms representing interaction with matter currents does not alter the topological nature of the action because the eikonal form of the source currents can also be written as boundary terms. Incorporating these terms, the $S$ matrix can be easily derived from the action in the saddle point approximation. The resulting scattering amplitude is the expression (7) for gravity and $G s \rightarrow-e e^{\prime}$ for electromagnetism. In a short while we shall see how both these terms can be incorporated in a single scattering amplitude formula. Finally, with the full Einstein-Maxwell action

$$
S=S_{E}+S_{\mathrm{EM}}=-\int d^{4} x \sqrt{-g}\left(\frac{R}{G}+\frac{1}{4} g^{\mu \rho} g^{\nu \lambda} F_{\mu \nu} F_{\rho \lambda}\right),
$$

the first (pure gravity) part once again reduces to the action on the boundary. For the second (electromagnetism coupled to gravity) part, the argument is more subtle. The results are best demonstrated in the units of Ref. [5], where it was assumed that $d x^{\mu}$ s were dimensionless, whereas $g_{\mu \nu}$ had dimensions $L^{2}, L$ signifying a length dimension. For dimensional consistency, the other relevant quantities are associated with the dimensions

$$
\begin{gathered}
\sqrt{-g} \sim L^{4}, \quad g^{\mu \nu} \sim L^{-2} ; \\
d^{4} x \sim 1, \quad x_{\mu} \sim L^{2} \\
\partial_{\mu} \sim 1, \quad \partial^{\mu} \sim L^{-2} ; \\
A^{\mu} \sim L^{-2}, \quad A_{\mu} \sim 1 ; \\
F_{\mu \nu} \sim 1, \quad F^{\mu \nu} \sim L^{-4} .
\end{gathered}
$$

Now let us consider the Maxwell action in an arbitrary space-time background:

$$
S_{\mathrm{EM}}=-\frac{1}{4} \int d^{4} x \sqrt{-g} F_{\mu \nu} F^{\mu \nu} .
$$

Splitting it up into the longitudinal, transverse, and the mixed parts, it takes the form

$$
S_{\mathrm{EM}}=-\frac{1}{4} \int d^{4} x \sqrt{-g}\left(F_{\alpha \beta} F^{\alpha \beta}+2 F_{\alpha i} F^{\alpha i}+F_{i j} F^{i j}\right) .
$$

Now we scale the longitudinal components of all the tensors by a small dimensionless parameter $\lambda \sim \sqrt{t / s}$, as

$$
\begin{gathered}
x_{\alpha} \rightarrow \lambda^{2} x_{\alpha} ; \\
F_{\mu \nu} \rightarrow F_{\mu \nu}, \quad F^{\alpha \beta} \rightarrow \lambda^{-4} F^{\alpha \beta}, \quad F^{\alpha i} \rightarrow \lambda^{-2} F^{\alpha i} ; \\
g_{\alpha \beta} \rightarrow \lambda^{2} g_{\alpha \beta}, \quad \sqrt{-g} \rightarrow \lambda^{2} \sqrt{-g} .
\end{gathered}
$$

Note that the transverse components remain unchanged. The rationale behind this scaling is that due to the high center-ofmass energy $\sqrt{s}$, the longitudinal length scales undergo a high Lorentz contraction which is incorporated in the smallness of the corresponding scaled quantities. The field components that survive after taking the limit $\lambda \rightarrow 0$ in the action are to be regarded as the only relevant degrees of freedom in the kinematical domain of interest. With this in mind, the scaled electromagnetic action is

$$
\begin{aligned}
S_{\mathrm{EM}} \rightarrow & -\frac{1}{4} \int d^{4} x \sqrt{-g} \lambda^{2}\left(\frac{1}{\lambda^{4}} F_{\alpha \beta} F^{\alpha \beta}\right. \\
& \left.+\frac{1}{\lambda^{2}} 2 F_{\alpha i} F^{\alpha i}+F_{i j} F^{i j}\right) .
\end{aligned}
$$

As in the case of flat space-time, the first term is highly oscillatory in the quantum partition function, which dictates the dominant modes to be

$$
F_{ \pm}=0
$$

admitting of the earlier solution

$$
A_{ \pm}=\partial_{ \pm} \Omega .
$$

As already mentioned, the transverse components of the gauge potential $A_{i}$ can be set to zero since they decouple; the reduced action is thus

$$
S_{\mathrm{EM}}=-\frac{1}{2} \int d^{4} x \sqrt{-g} F_{\alpha i} F^{\alpha i} .
$$

Now, as pointed out after Eq. (18), the metric $g_{\alpha \beta}$ is conformally flat in the longitudinal subspace, so that the conformally invariant quantity $\sqrt{-g} g^{\alpha \beta}$ can be transformed into the longitudinal Minkowski metric $\eta^{\alpha \beta}$ by local variations of $X^{a}$. Consequently, using Eq. (18) we can write

$$
S_{\mathrm{EM}}=-\frac{1}{2} \int d^{2} x_{\perp} \sqrt{h} h^{i j} \int d x^{+} d x^{-} F_{\alpha i} F_{j}^{\alpha} .
$$

On substituting the constraints (20),

$$
S_{\mathrm{EM}}=\frac{1}{2} \int d^{2} x_{\perp} \sqrt{h} h^{i j} \int d x^{+} d x^{-} \partial_{i} \partial_{\alpha} \Omega \partial_{j} \partial^{\alpha} \Omega .
$$

As before, in the Lorentz gauge, this reduces to the action (19) for Minkowski space scattering which enforces $h_{i j}=\delta_{i j}$.

In summary, the Einstein-Maxwell action in totality reduces to two separate terms, representing the gravity and electromagnetic interactions, respectively:

$$
S_{E}+S_{\mathrm{EM}} \rightarrow S_{E[\partial M]}+S_{\mathrm{EM}[\partial M]} .
$$

Thus, the $S$ matrix calculated from the total boundary action will just be an incoherent superposition of the individual $S$ matrices. This is the statement of decoupling that was sought. For completeness, we give the expression for the scattering amplitude of two point particles with charges $e$ and $e^{\prime}$ interacting via gravity and electromagnetism [9] : 


$$
f(s, t)=\frac{1}{t} \frac{\Gamma\left(1-i G s+i e e^{\prime}\right)}{\Gamma\left(1+i G s-i e e^{\prime}\right)}\left(\frac{1}{-t}\right)^{-i G s+i e e^{\prime}},
$$

In effect, this means that we can replace the gravitational "coupling" $G s$ by the effective coupling constant $G s-e e^{\prime}$ in the presence of electromagnetism. It is remarkable that this decoupling is manifest already at the level of the action, once the kinematical restrictions are imposed on it.

We now move on to dilaton gravity. The action that we must consider is (in the Einstein metric)

$$
S_{D}=\int d^{4} x \sqrt{-g}\left(-\frac{R}{G}+e^{-2 \phi} F_{\mu \nu} F^{\mu \nu}+2 \partial_{\mu} \phi \partial^{\mu} \phi\right) .
$$

The first term is identical to the general relativity action and independent of the dilaton field, yielding Eq. (17) once again. However, the interaction term involving the MaxwellEinstein-dilaton fields is no longer amenable to earlier simplifications. Although the scaling arguments will still hold, the counterparts of Eqs. (25) and (26) are, respectively,

$$
S_{\mathrm{EM}}=-\frac{1}{2} \int d^{4} x \sqrt{-g} e^{-2 \phi} F_{\alpha i} F^{\alpha i}
$$

and

$$
S_{\mathrm{EM}}=-\frac{1}{2} \int d^{2} x_{\perp} \sqrt{h} h^{i j} \int d x^{+} d x^{-} e^{-2 \phi} F_{\alpha i} F_{j}^{\alpha} .
$$

The constraint $F_{ \pm}=0$ will remain unchanged along with its solution $A_{ \pm}=\partial_{ \pm} \Omega$. As before, $A_{i}$ is taken to be zero. Thus the above equation becomes

$$
\begin{aligned}
S_{\mathrm{EM}}= & -\frac{1}{2} \int d^{2} x_{\perp} \sqrt{h} h^{i j} \int d x^{+} d x^{-}\left\{\partial_{\alpha}\left[e^{-2 \phi}\left(\partial_{i} \Omega\right)\left(\partial_{j} \partial^{\alpha} \Omega\right)\right]\right. \\
& \left.-e^{-2 \phi}\left(\partial_{i} \Omega\right)\left(\partial_{j} \partial_{\alpha} \partial^{\alpha} \Omega\right)+e^{-2 \phi}\left(\partial_{i} \Omega\right)\left(\partial_{j} \partial^{\alpha} \Omega\right) \partial_{\alpha} \phi\right\} .
\end{aligned}
$$

The first term is a total divergence and hence can be converted into a boundary term. The second term can be made to vanish by virtue of the Lorentz gauge condition. The new significant piece is the last term, which is a "bulk" piece, dependent on the local field coordinates. This term can neither be made to vanish, nor be transferred to the boundary $\partial M$ for generic values of the dilaton field. Thus, the local degrees of freedom fail to decouple from the theory and eikonal approximation techniques used to calculate the $S$-matrix can no longer be employed. These conclusions are of course not dependent on the choice of coordinates. In terms of the string metric, the dilaton couples to the scalar curvature as well as the gauge fields. Thus, in this case, both the terms in the action would fail to give pure boundary terms.

As in the previous sections, it is natural to investigate the status of the above analysis in the extremal limit. However, here since we are dealing with the action and not with the solutions, it is not clear as to how one can implement the extremality condition. Note however that the bulk term disappears for dilaton configurations that are independent of the null coordinates, i.e., when the dilaton ceases to be a propagating degree of freedom. For example, consider the extremal limit of the black hole solution. The solution for the dilaton field, derived from the action (29) is

$$
e^{2 \phi}=e^{2 \phi_{0}}\left(1-\frac{\alpha}{M r}\right)
$$

The extremality condition simplifies this to

$$
e^{2 \phi}=e^{2 \phi_{0}}\left(1-\frac{2 G M}{r}\right) .
$$

Now, the eikonal limit requires that we take the particle masses to be vanishingly small. Hence, on taking $M \rightarrow 0$ in the above equation, we see that $\phi$ approaches its constant asymptotic value identically. Thus the extremal dilaton solution certainly is sufficient since the dilaton field is frozen at its extremal value; however, it appears to be a bit of an overkill, since all one needs to eliminate the bulk term is a dilaton field depending only on the transverse coordinates.

\section{RESUMMATION OF LADDER EXCHANGES}

Historically, the earliest approach to the eikonal approximation in relativistic field theory entailed analyses of an infinite set of ladder-type exchange Feynman graphs in which the momenta of the external lines are assumed to remain more or less fixed on-shell, so that virtual particles carried almost no momenta [15]. The motivation behind this restriction is the assumption that in the high energy limit, there are well-defined classical trajectories for the particles, which deviate only slightly from free particle trajectories. Ignoring standard radiative corrections, the infinite sum is seen to admit [15] a closed form expression, which indeed captures the leading behavior of the scattering amplitudes for high centerof-mass energies. A similar eikonal resummation for linearized gravity, involving ladder exchange of gravitons, was performed in Ref. [16], which reproduced the quantum mechanical result (7). The Feynman rules were derived from the following linearized gravity action:

$$
\begin{aligned}
S_{\mathrm{LG}}= & \frac{1}{G} \int d^{4} x \frac{1}{8} h_{\mu \nu}\left(\eta^{\mu \lambda} \eta^{\nu \sigma}+\eta^{\mu \sigma} \eta^{\nu \lambda}-\eta^{\mu \nu} \eta^{\lambda \sigma}\right) \square h_{\lambda \sigma} \\
& +\frac{1}{2} \chi \square \chi+\frac{1}{2} h_{\mu \nu}\left(\partial^{\mu} \chi \partial^{\nu} \chi-\frac{1}{2} \eta^{\mu \nu} \partial_{\sigma} \chi \partial^{\sigma} \chi\right),
\end{aligned}
$$

where the metric has been linearized as $g_{\mu \nu}=\eta_{\mu \nu}+h_{\mu \nu}$. The scalar field $\chi$ corresponds to the particles undergoing scattering. The eikonal amplitude obtained in this case, for nonvanishing masses, is given by [16]

$$
i \mathcal{M}(s, t) \sim \frac{\sqrt{s\left(s-4 m^{2}\right)}}{t} \frac{\Gamma(1-i \alpha(s))}{\Gamma(i \alpha(s))},
$$

where,

$$
\alpha(s)=G \frac{\left(s-2 m^{2}\right)^{2}-2 m^{4}}{\sqrt{s\left(s-4 m^{2}\right)}} .
$$

For $m=0$, this reduces to Eq. (7). 
In the dilaton gravity case, if we start with the dilaton gravity action coupled to the matter field $\chi$ in the string metric,

$$
S=\int d^{4} x \sqrt{-g} e^{-2 \phi}\left(-\frac{R}{G}-4 \partial_{\mu} \phi \partial^{\mu} \phi+F^{2}-\frac{1}{2} \partial_{\mu} \chi \partial^{\mu} \chi\right),
$$

then the condition of the existence of the classical trajectory of the test particles appears invalidated, since as already mentioned, for small particle masses, the space-time singularity at $r=\alpha / M$ spreads indefinitely and traps any other test particle at arbitrarily large impact parameters. Thus an eikonal graph calculation with the above action is seemingly fraught with pitfalls. Despite these, we proceed with linearizing the dilaton field, as was done for the metric tensor. We write $\phi$ in the form

$$
\phi=\phi_{0}+f
$$

where $f$ represents the small quantum fluctuations around the constant asymptotic value $\phi_{0}$. Before embarking on perturbative calculations with this simplified action, a heuristic justification of this linearization may be given as follows. A rough estimate of the magnitude of $f$ can be made from the classical solution (33):

$$
f \approx\left|\phi-\phi_{0}\right| \sim\left|\ln \left(1-\frac{\alpha}{M r}\right)\right| .
$$

Demanding this to be small leads to the condition

$$
\left|1-\frac{\alpha}{M r}\right| \approx 1 \Leftrightarrow\left|\frac{\alpha}{M r}\right| \approx 0,
$$

for arbitrary $r$. This of course means that $\alpha$ should approach zero at least as $M^{2}$, which is the extremality condition. Hence a linearized approximation seems reasonable in the extremal limit.

To leading orders in the graviton and dilaton fluctuations, the dilaton gravity action now becomes

$$
\begin{aligned}
S= & \frac{e^{-2 \phi_{0}}}{G} \int d^{4} x(1-2 f) \frac{1}{8} h_{\mu \nu}\left(\eta^{\mu \lambda} \eta^{\nu \sigma}+\eta^{\mu \sigma} \eta^{\nu \lambda}\right. \\
& \left.-\eta^{\mu \nu} \eta^{\lambda \sigma}\right) \square h_{\lambda \sigma}-e^{-2 \phi_{0}} \int d^{4} x\left(1+\frac{1}{2} h_{\alpha}^{\alpha}\right)(1-2 f) \\
& \times\left(-4 \partial_{\mu} f \partial^{\mu} f+F^{2}+\partial_{\mu} \chi \partial^{\mu} \chi\right) .
\end{aligned}
$$

Since the graviton and photon ladder summations are known, we concentrate on the dilaton-matter field interactions, given by the last term. The new momentum dependent $(\chi-\chi-f)$ vertex is associated with the factor $-2 p \cdot p^{\prime}$, where $p$ and $p^{\prime}$ are the momenta associated with the two $\chi$ lines. They give rise to an infinite set of ladders with intermediate dilaton exchanges. Since these can be summed in a fairly straightforward manner, we simply give a schematic derivation of the final result. The Born amplitude (corresponding to a single dilaton exchange) is

$$
i \mathcal{M}_{\mathrm{Born}}=\frac{i p_{1}^{2} p_{2}^{2}}{\left(p_{1}-p_{3}\right)^{2}-i \epsilon} .
$$

Here, $p_{1}$ and $p_{2}$ are the incoming and $p_{3}$ and $p_{4}$ are the outgoing four-momenta. They are related by the constraint $p_{1}+p_{2}-p_{3}-p_{4}=0$. For the next higher order ladder, there are four distinct diagrams depending on the momentum labels for the two exchanged particles. Using the eikonal form of the external matter propagators $[15,16]$, namely,

$$
\frac{1}{(p+k)^{2}+m^{2}-i \epsilon} \approx \frac{1}{2 p \cdot k-i \epsilon},
$$

the one loop amplitude is

$$
\begin{aligned}
p_{1}^{4} p_{2}^{4} \int & \frac{d^{4} k}{(2 \pi)^{4}} \frac{1}{k^{2}-i \epsilon} \frac{1}{\left(p_{1}-p_{3}-k\right)^{2}-i \epsilon} \\
& \times \frac{1}{2}\left(\frac{1}{-2 p_{1} \cdot k-i \epsilon} \frac{1}{2 p_{2} \cdot k-i \epsilon}\right. \\
+ & \frac{1}{-2 p_{1} \cdot k-i \epsilon} \frac{1}{-2 p_{4} \cdot k-i \epsilon} \\
+ & \left.\frac{1}{2 p_{3} \cdot k-i \epsilon} \frac{1}{2 p_{2} \cdot k-i \epsilon}+\frac{1}{2 p_{3} \cdot k-i \epsilon} \frac{1}{-2 p_{4} \cdot k-i \epsilon}\right) .
\end{aligned}
$$

By doing the combinatorics carefully, it can be shown that the infinite set of ladders exponentiate to give the final amplitude as

$$
i \mathcal{M}=-p_{1}^{2} p_{2}^{2} \int d^{4} x e^{-\left(p_{1}-p_{3}\right) \cdot x} \Delta(x) \frac{e^{i \psi}-1}{\psi},
$$

where $\Delta(x)$ is the Fourier transform of the dilaton propagator and

$$
\begin{aligned}
\psi= & -p_{1}^{2} p_{2}^{2} \int \frac{d^{4} k}{(2 \pi)^{4}} e^{i k \cdot x} \frac{1}{k^{2}-i \epsilon}\left(\frac{1}{-2 p_{1} \cdot k-i \epsilon} \frac{1}{2 p_{2} \cdot k-i \epsilon}\right. \\
& +\frac{1}{-2 p_{1} \cdot k-i \epsilon} \frac{1}{-2 p_{4} \cdot k-i \epsilon}+\frac{1}{2 p_{3} \cdot k-i \epsilon} \frac{1}{2 p_{2} \cdot k-i \epsilon} \\
& \left.+\frac{1}{2 p_{3} \cdot k-i \epsilon} \frac{1}{-2 p_{4} \cdot k-i \epsilon}\right) .
\end{aligned}
$$

Assuming small momentum transfers, we can take $p_{1} \approx p_{3}$ and $p_{2} \approx p_{4}$, to obtain

$$
\psi=-\frac{p_{1}^{2} p_{2}^{2}}{16 \pi E p} \ln \mu x_{\perp} .
$$

Here $x_{\perp}$ is the transverse coordinate, $(E, \pm p)$ are the fourmomentum vectors of the two particles in the center-of-mass frame and $\mu$ is an irrelevant mass parameter. With this, the explicit evaluation of $\mathcal{M}$ in Eq. (41) leads to

$$
i \mathcal{M}=\frac{i p_{1}^{2} p_{2}^{2}}{-t} \frac{\Gamma\left(1-i p_{1}^{2} p_{2}^{2} / 32 \pi E p\right)}{\Gamma\left(1+i p_{1}^{2} p_{2}^{2} / 32 \pi E p\right)}\left(\frac{4 \mu^{2}}{-t}\right)^{-i\left(p_{1}^{2} p_{2}^{2} / 32 \pi E p\right)}
$$

where $-t$ is the square of the momentum transfer. Now, plugging in the on-shell conditions $p_{1}^{2}, p_{2}^{2}=m^{2}$, the above amplitude decays to zero for vanishing particle masses. This means that these ladders do not contribute to the scattering 
amplitude at all. Thus we are left with the original set of matter-graviton and matter-photon ladder diagrams of Refs. $[16,6]$ and the corresponding finite scattering amplitude for Einstein-Maxwell theory (28).

It now seems that the pathologies that we had encountered earlier have disappeared. Note however that the preceding results would only hold when the dilaton fluctuations are small enough for linearization to go through, i.e., $\left|\phi-\phi_{0}\right| \ll 1$ (in Planck units). Now, in the low energy limit of string theory, the string coupling parameter $g_{s}$ is usually related to the asymptotic value of the dilaton, $g_{s} \equiv \exp \left(\phi_{0}\right)$. In the regime of perturbative string theory one must have $g_{s} \ll 1$, which implies that $\phi_{0}$ itself should be large and negative (in Planck units), i.e., $\left|\phi_{0}\right| \gg 1$. It is not clear that these dual requirements are compatible. Thus, our linearization of the dilaton gravity action may not correspond to the perturbative domain of string theory. But if we now relax this restriction to include large $g_{s}$ regimes, then the linearization is perfectly justified and there is no problem with resummation of dilatonic ladder exchanges. Since certain extremal black hole solutions of string theory [17] have been advertized as exact quantum states not subject to the perturbative restriction $g_{s} \ll 1$, it is perhaps not surprising that Planckian scattering of point particles, which is inherently nonperturbative in nature, is reasonable only outside the perturbative regime of string theory.

\section{CONCLUSION}

We begin this section with a survey of our principal findings. The curvature singularity away from the origin in the nonextremal charged dilaton black hole metric is shown to be responsible for the absence of a plane-fronted gravitational shock wave, when such a black hole is Lorentzboosted to luminal velocities. Instead of a single plane ( $x^{-}=0$ in the Schwarzschild case), the singular geometry in the Planckian eikonal limit consists of a three-dimensional region whose thickness is proportional to the dilatonic charge $\alpha \equiv Q^{2} \exp \left(-\phi_{0}\right)$. Consequently, Planckian scattering amplitudes in this model can no longer be computed using the simple techniques of Ref. [1]. The problem resurfaces in the external metric approach in that the radial component of the particle equation of motion does not reduce to a Schrödinger-like equation in the eikonal approximation. In fact, the discontinuities in the coefficients of this equation in the relevant kinematical limit render the equation unsolvable. Remarkably, in both approaches, the malady disappears upon imposing the extremal limit; in the first (heuristic) approach, the dilaton charge simply shrinks to zero upon boosting, thereby yielding the same plane-fronted gravitational shock wave as in the Schwarzschild case. An identical situation ensues in the external metric formalism, where the discontinuities previously preventing the solution of the quantum equation of motion are now gone. Since the static extremal dilatonic black hole metric looks quite different from the Schwarzschild metric, the end result is a pleasant surprise.

The alternative approach involving identification of the degrees of freedom participating in eikonal scattering and an effective field theory of these degrees of freedom contained in Ref. [5] has also been pursued for the dilaton gravity action. Indeed, unlike in the case of the Einstein-Hilbert and Maxwell actions, this action does not reduce in the appropriate scaling limit to a "boundary" field theory. The offending terms disappear for nonpropagating dilaton configurations such as would appear for extremal black hole solutions in the massless limit. The situation is, however, quite different for the standard field theoretic approach to the eikonal of summing ladder exchange Feynman graphs. In this case, a linearized approximation to the dilaton gravity action, retaining terms only up to quadratic in the dilaton field, does indeed yield a summed amplitude of ladders and crossed ladders in a closed form in the eikonal kinematical domain. The problem shows up in a rather subtle manner: the restriction on the asymptotic value of the dilaton field from string perturbation theory is not compatible with the requirement of small dilaton fluctuations around the asymptotic value necessary for linearization of the action (and the subsequent derivation of the eikonal amplitude).

The above analyses point unambiguously to the fact that extremal black holes play a very special role in eikonal scattering. Recall that our motivation to consider dilaton gravity was to model charged point particles as sources of the dilaton gravity metric instead of the canonical ReissnerNordström metric. The reason was of course that the low energy string equations of motion naturally give rise to the former. However, this modeling seems to work only in the extremal limit. Perhaps this is the manner in which string theory, which gives rise inexorably to dilaton gravity at low energies as an effective theory of gravitation, also cures the problems that go with it. The central role played by extremal black holes is emphasized time and again in recent literature on duality because of the strong possibility of their being elementary string excitations [17]. Our work stresses this further in terms of nonperturbative behavior in the eikonal limit.
[1] G. 't Hooft, Phys. Lett. B 198, 61 (1987); Nucl. Phys. B304, 867 (1988).

[2] D. Amati, M. Ciafaloni, and G. Veneziano, Phys. Lett. B 197, 81 (1987); Int. J. Mod. Phys. A 3, 1615 (1988); Nucl. Phys. B347, 550 (1990).

[3] P. Aichelburg and R. Sexl, Gen. Relativ. Gravit. 2, 303 (1971).

[4] T. Dray and G. 't Hooft, Nucl. Phys. B253, 173 (1985).

[5] H. Verlinde and E. Verlinde, Nucl. Phys. B371, 246 (1992).

[6] R. Jackiw, D. Kabat, and M. Ortiz, Phys. Lett. B 277, 148 (1992).
[7] S. Das and P. Majumdar, Phys. Rev. Lett. 72, 2524 (1994).

[8] S. Das and P. Majumdar, Phys. Lett. B 348, 349 (1995).

[9] S. Das and P. Majumdar, Phys. Rev. D 51, 5664 (1995).

[10] S. Das and P. Majumdar, IMSc Report No. 95/8, hep-th/ 9504060, 1995 (unpublished).

[11] M. B. Green, J. H. Schwarz, and E. Witten, Superstring Theory (Cambridge University Press, Cambridge, England, 1987), Vol. 1, p. 180.

[12] H. Garfinkle, G. Horowitz, and A. Strominger, Phys. Rev. D 43, 3140 (1991); 45, 3888(E) (1992). 
[13] E. L. Ince, Ordinary Differential Equations (Dover, New York, 1956), p. 73.

[14] A. S. Davydov, Quantum Mechanics, 2nd ed. (Pergamon, New York, 1965), p. 482.

[15] H. D. I. Abarbanel and C. Itzykson, Phys. Rev. Lett. 23, 53
(1969); M. Lévy and J. Sucher, Phys. Rev. 186, 1656 (1969). [16] D. Kabat and M. Ortiz, Nucl. Phys. B388, 570 (1992).

[17] A. Sen, Mod. Phys. Lett. A 10, 2081 (1995), and references therein. 